\section{Structure, odd lines and topological entropy of disorder of amorphous silicon. Correction}

\section{Frederick Wooten}

Department of Applied Science, University of California, Davis, CA 95616, USA Correspondence e-mail: fowooten@ucdavis.edu

Wooten [Acta Cryst. (2002), A58, 346-351] defines cells in amorphous $\mathrm{Si}$ as being bounded by irreducible rings of atoms, which can be viewed as distorted polygons. These irreducible rings are, roughly speaking, shortest rings. However, the definition used is incomplete and leads to some large rings being counted as irreducible when close examination reveals they are not. In particular, the incomplete definition counts four ninefold rings (out of a total of 1041 ninefold rings) as being irreducible. Yet a detailed examination reveals that these four rings bound a set of smaller rings, and are clearly not irreducible in any meaningful sense of the word. An extended definition has been given [Rivier \& Wooten (2003). MATCH - Commun. Math. Comput. Chem. 48, 145-153], and described at length, that removes the difficulties. It results in a small increase in entropy, approaching slightly closer to the ideal. The first paragraph of Wooten (2002) incorrectly states that the nearest-neighbor distance in $\mathrm{Si}$ is $3.5 \AA$, rather than the correct value of $2.35 \AA$. This misprint has no effect on anything else.

\section{References}

Rivier, N. \& Wooten, F. (2003). MATCH - Commun. Math. Comput. Chem. 48, 145-153.

Wooten, F. (2002). Acta Cryst. A58, 346-351. 